\title{
High-speed and low-distortion solution for time-correlated single photon counting measurements: A theoretical analysis
}

A. Cominelli, G. Acconcia, P. Peronio, M. Ghioni, and I. Rech

Citation: Review of Scientific Instruments 88, 123701 (2017); doi: 10.1063/1.4996690

View online: https://doi.org/10.1063/1.4996690

View Table of Contents: http://aip.scitation.org/toc/rsi/88/12

Published by the American Institute of Physics

\section{Articles you may be interested in}

Effects of transients in LIGO suspensions on searches for gravitational waves

Review of Scientific Instruments 88, 124501 (2017); 10.1063/1.5000264

Digitally controlled analog proportional-integral-derivative (PID) controller for high-speed scanning probe microscopy

Review of Scientific Instruments 88, 123712 (2017); 10.1063/1.5010181

Phase sensitive imaging of $10 \mathrm{GHz}$ vibrations in an AIN microdisk resonator

Review of Scientific Instruments 88, 123709 (2017); 10.1063/1.4995008

The Oxford space environment goniometer: A new experimental setup for making directional emissivity measurements under a simulated space environment

Review of Scientific Instruments 88, 124502 (2017); 10.1063/1.4986657

A high-resolution combined scanning laser and widefield polarizing microscope for imaging at temperatures from $4 \mathrm{~K}$ to $300 \mathrm{~K}$

Review of Scientific Instruments 88, 123705 (2017); 10.1063/1.5009529

Experimental confirmation of the atomic force microscope cantilever stiffness tilt correction

Review of Scientific Instruments 88, 123710 (2017); 10.1063/1.4986201

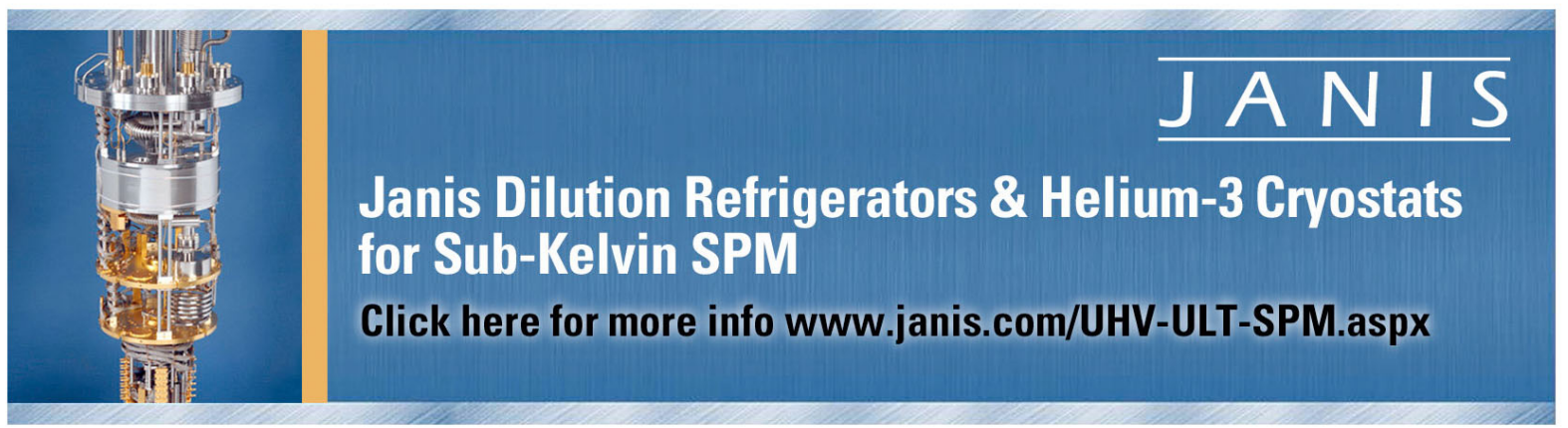




\title{
High-speed and low-distortion solution for time-correlated single photon counting measurements: A theoretical analysis
}

\author{
A. Cominelli, ${ }^{\text {a) }}$ G. Acconcia, P. Peronio, M. Ghioni, and I. Rech \\ Politecnico di Milano, Dipartimento di Elettronica, Informazione e Bioingegneria (DEIB), \\ Piazza Leonardo da Vinci 32, 20133 Milano, Italy
}

(Received 18 July 2017; accepted 8 November 2017; published online 4 December 2017)

\begin{abstract}
In this paper, we describe a novel solution to increase the speed of Time-Correlated Single Photon Counting (TCSPC) measurements by almost an order of magnitude while providing, in principle, zero distortion regardless of the experimental conditions. Typically, the relatively long dead time associated with the conversion electronics requires a proper tune of the excitation power in order to avoid distortions of the reconstructed waveform due to pileup and counting loss. As a result, the maximum operating rate of a TCSPC channel is now limited between $1 \%$ and $5 \%$ of the excitation frequency, thus leading to relatively long acquisition times. We show that negligible distortion (below 1\%) is guaranteed if the dead time associated with the converter is kept below the dead time of the detector, and at the same time the detector dead time is matched to the duration of the excitation period. In this way, unprecedented high-speed operation is possible. In this paper, we provide a theoretical analysis of the technique, including the main non-idealities which are introduced by a generic physical implementation. The results are supported by both numerical simulations and analytical calculations. Published by AIP Publishing. https://doi.org/10.1063/1.4996690
\end{abstract}

\section{INTRODUCTION}

Time-Correlated Single Photon Counting (TCSPC) is a powerful tool to estimate fluorescence lifetimes. In life science, for instance, TCSPC allows the highest time resolution in Fluorescence Lifetime Imaging Microscopy (FLIM) and Förster resonance energy transfer experiments. ${ }^{1-4}$

TCSPC basically consists in the periodical excitation of a sample with a pulsed laser and in the record of the time of arrivals of the re-emitted photons. Currently available acquisition systems can detect only one photon per excitation cycle; if more than one photon impinges on the detector during a period, the system undergoes the so-called pileup effect and the reconstructed waveform is distorted. ${ }^{5}$ In addition, counting loss due to the finite speed of the acquisition system can lead not only to a counting efficiency drop of the system, but it is also another potential source of distortion. ${ }^{5}$ In order to avoid both the pileup and counting-loss artefacts, the power of the excitation source can be adjusted to limit the number of impinging photons in a period to far less than one (typical values range between 0.01 and 0.05$).^{5,6}$ It follows that a relatively high number of excitation cycles, so a long measurement time are needed to accumulate a statistically relevant number of events.

Recently, much effort has been done to decrease the dead time associated with a TCSPC acquisition chain. On one hand, extremely fast converters have been developed, ${ }^{7-10}$ with a dead time in the order of few nanoseconds. On the other hand, the exploitation of hybrid photomultipliers has led to a reduction of the detector dead time down to about $1 \mathrm{~ns},{ }^{11}$ thus increasing its counting capability, but at the expense of a significant distortion at high-rate operation. ${ }^{11}$

a) alessandro.cominelli@polimi.it
A different approach consists in the parallelization of $\mathrm{N}$ independent acquisition chains, which permit, in principle, to increase the counting capability of the system by a factor $\mathrm{N}{ }^{6}$ To this aim, many multichannel solutions based on SinglePhoton Avalanche Diode (SPAD) arrays have been proposed in the literature. ${ }^{12-16}$ Nevertheless, the exploitation of a large number of acquisition chains demands for complicated readout architectures to cope with a limited bandwidth toward the external elaboration unit, ${ }^{17}$ and large arrays proposed so far do not guarantee a measurement speed proportional to the number of channels, thus limiting the advantages of having a high degree of parallelism. ${ }^{17}$ At the same time, large integrated arrays suffer from a trade-off between the number of channels and performance of the system, in terms of precision and linearity. ${ }^{12,13,15}$

In this paper, we present a novel solution aimed at maximizing the operation speed of a single channel while keeping the distortion under an acceptable level. Our solution is made feasible by the recently proposed fast time-measurement circuits, ${ }^{7-10}$ which feature a negligible dead time, thus limiting counting loss artefacts, and recent active quenching circuits for SPADs, which permit a fine tune of the detector dead time. ${ }^{18,19}$ In this scenario, we propose a dead time optimization technique. In particular, we show that a system operating with a dead time matched to an integer number of excitation periods opens the way to a remarkable increase of the measurement speed up to a factor of 8 while keeping the distortion well below 1\%. It is worth highlighting that our solution can be easily extended to a multichannel approach to further increase the measurement speed of the system.

The paper is organized as follows: first of all, a brief description of pileup and counting loss effects is given in Sec. II for a typical TCSPC measurement channel which 
features a relatively high conversion dead time. The introduction of a fast time-measurement electronics leads to a completely different scenario, which is described in Sec. III. Here an optimum working condition is found, which allows us to minimize the distortion of the recorded curve. The proposed solution is analyzed by means of analytical calculations, fully supported by numerical simulations. Then, in Sec. IV, we analyze the effects of the major non-idealities which can affect a physical implementation of the solution, namely, a jitter superimposed on the duration of the dead time and the influence of a finite reset time to restore the biasing condition of the detector at the end of the dead time. Conclusions are drawn in Sec. V.

\section{PILEUP AND COUNTING LOSS}

A TCSPC measurement basically consists in the reconstruction of the fluorescence decay from the probability density function (pdf) of the single-photons time of arrival on the detector. In the simple case of fluorescence decay with a single time constant, the pdf is proportional to the following expression:

$$
P_{\text {imp }}(t)=P_{0} \cdot e^{-t / \tau} \cdot u(t),
$$

where $P_{\text {imp }}(t) d t$ is the mean number of photons that impinge on the detector during an infinitesimal-sized interval $d t$ centered at time $t$ within the excitation period, $\tau$ is the decay time constant, $u(t)$ is the Heaviside step function, and $P_{0}$ is a constant proportional to the power of the luminous signal.

In an ideal TCSPC system, every event is accumulated in a histogram, which is proportional to $P_{i m p}(t)$. Conversely, in a real TCSPC acquisition system, two phenomena can lead to loss of events: classic pileup and counting loss due to a non-zero dead time of both the detector and the conversion electronics. $^{5}$

The classic pileup is due to the fact that TCSPC acquisition systems can typically record only a single event per excitation cycle; so early photons experience a higher recording probability, leading to an artificial reduction of the decay time constant in the recorded curve. The pileup distortion increases with the average number of impinging photons in a period, $P$. In particular, for $P$ well below unity, the measured time constant $\tau_{\text {meas }}$ can be expressed as follows: ${ }^{5}$

$$
\tau_{\text {meas }} \approx\left(1-\frac{P}{4}\right) \cdot \tau \text {. }
$$

In order to limit distortion, $P$ is usually adjusted between 0.01 and 0.05 ; in this way, the estimation error is limited to about $1.25 \%$.

The pileup model properly describes the systems exploiting the detector and electronics with a negligible dead time, either operated in nonreversed or reversed start-stop. ${ }^{5}$ Nevertheless, in real TCSPC experiments, the system remains blind for a fixed time interval after an event has been measured. This effect leads to additional counting loss and represents the main source of efficiency drop in real TCSPC systems, giving rise to a nonlinear increase of the average number of recorded events in a period as a function of $P .^{5}$

\section{DETECTOR DEAD TIME AND DISTORTION MINIMIZATION}

In TCSPC experiments, the dead time of the conversion electronics usually represents the main limitation to the measurement speed. Nevertheless some fast conversion circuits have been proposed in recent years, providing a dead time in the order of few nanoseconds. ${ }^{7-9}$ For instance, in 2015, we proposed a fast time-measurement circuit, ${ }^{10}$ which is based on Time-to-Amplitude Converters (TACs). It guarantees a negligible dead time, along with high performance, that is a Differential Non-Linearity (DNL) of about $2 \%$ peak to peak and a timing jitter as low as 55 ps FWHM. ${ }^{10}$

In systems exploiting a fast converter, the detector dead time represents the only source of counting loss. In this paper, we demonstrate the existence of an optimum dead time value, which allows us to minimize distortion, irrespective of the value of the decay time constant, thus paving the way for highspeed TCSPC experiments using a single acquisition channel. In particular, we show that, irrespective of the rate of impinging photons, the distortion is exactly zero if the dead time is equal to an integer number of laser periods. In principle, the excitation period can be adjusted to reach this condition, but a fine tune of the laser frequency is rarely feasible. On the other hand, the dead time of the detector can be adjusted to match the excitation period. For instance, recently proposed Active Quenching Circuits (AQCs) coupled to SPAD detectors feature a tunable dead time, ${ }^{18,19}$ thus opening the way to dead time optimization.

In Fig. 1, the detector-related counting loss mechanism is shown for a system where the time-measurement circuitry does not introduce any appreciable loss of events. In this case, the dead time can end at any time instant of a subsequent period, depending on the arrival time of the recorded event, and both distortion efficiency and counting efficiency depend on the distribution of impinging photons over time, i.e., on the fluorescence lifetime $\tau$. This situation is analyzed in detail in Secs. III A-III C.

\section{A. Distortion}

In order to evaluate the distortion caused by the detector dead time, numerical simulations of the mechanism shown in Fig. 1 have been performed for different values of the dead time. In particular, a Poisson process has been used to simulate exponential pulses with a repetition period of $12.5 \mathrm{~ns}$, considering different values of decay time constant. Then the recorded events have been accumulated to form a histogram,

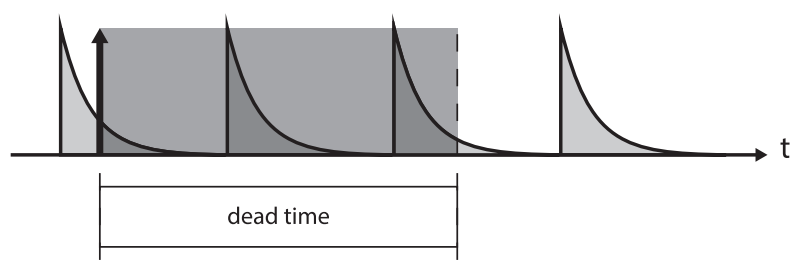

FIG. 1. Loss of events due to the detector dead time. Each time the system records an event, it remains blind for a fixed time interval. Exponential pulses represent $P_{i m p}(t)$, while the arrow highlights the photon arrival time. 


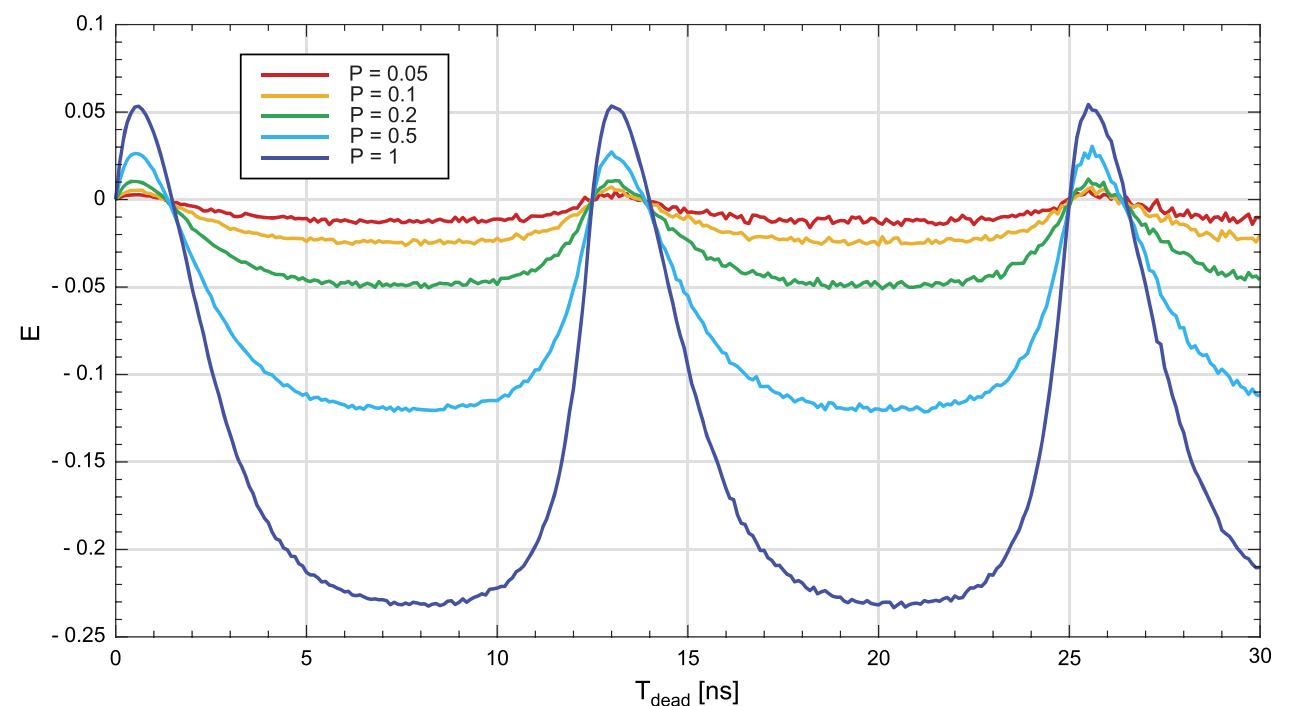

FIG. 2. Fractional error in the estimation of the time constant, $E$, as a function of the detector dead time $T_{\text {dead }}$. The situation of Fig. 1 has been simulated using fluorescence pulses with $\tau$ equal to $1 \mathrm{~ns}$ and laser period equal to $12.5 \mathrm{~ns}$. Then, a single time constant $\tau_{\text {meas }}$ has been estimated for the recorded histograms, using a center-of-mass method, and $E$ has been calculated. The result is shown for different values of $P$, that is, the average number of photons impinging on the detector during a period. considering a state-of-the-art resolution of $10 \mathrm{ps.} \mathrm{Finally,} \mathrm{a} \mathrm{sin-}$ gle time constant, $\tau_{\text {meas }}$, has been extracted from the recorded histograms using a center of mass method, ${ }^{20}$

$$
\tau_{\text {meas }}=\frac{\int_{0}^{T_{\text {laser }}} t \cdot h(t) \cdot d t}{\int_{0}^{T_{\text {laser }}} h(t) \cdot d t},
$$

where $h(t)$ is the recorded curve at the end of the measurement, while $T_{\text {laser }}$ is the excitation period.

It should be noted that expression (3) holds only if the duration of the fluorescence pulse is limited well below an excitation period, i.e., $\tau$ is far lower than $T_{\text {laser }}$.

Starting from the extracted lifetime, $\tau_{\text {meas }}$, the fractional estimation error $E$ has been calculated as follows:

$$
E=\frac{\tau_{\text {meas }}-\tau}{\tau} \text {. }
$$

The result of a simulation is shown in Fig. 2, where a fluorescence time constant of $1 \mathrm{~ns}$ has been considered, while the average number of impinging photons in a period, $P$, is used as a parameter. A relatively long measurement time of about $10 \mathrm{~s}$, which corresponds to about one billion excitation cycles, has been considered to accumulate a statistically relevant number of events in each histogram. In this way, it was possible to decrease the noise contribution due to Poisson statistics down to negligible values, ${ }^{21}$ in order to concentrate the analysis on the distortion introduced on the average recorded curves.

It is evident that the average estimation error is a periodic function of the dead time, whose period is equal to $12.5 \mathrm{~ns}$, that is, the duration of an excitation cycle, so the system features zero distortion at integer multiples of the excitation period. In this scenario, matching the detector dead time with the excitation period permits to surpass the pileup limit.

When there is no dead time $\left(T_{\text {dead }}=0\right)$, the system experiences no counting loss and the histogram $h(t)$ is proportional to the pulse shape expressed in (1), meaning that the fractional error $E$ is equal to zero. By increasing the dead time, the error moves to positive values, up to a local maximum (see Fig. 2). In this region, the dead time is lower than the duration of the fluorescence pulse, so a photon detected in the first part of the period does not mask the whole fluorescence signal. The result is a reduction of the recording probability in the central part of the pulse, as depicted in Fig. 3(a). It has been demonstrated that this kind of distortion leads to an overestimation of the time constant, hence to a positive value of $E .^{6}$ a)

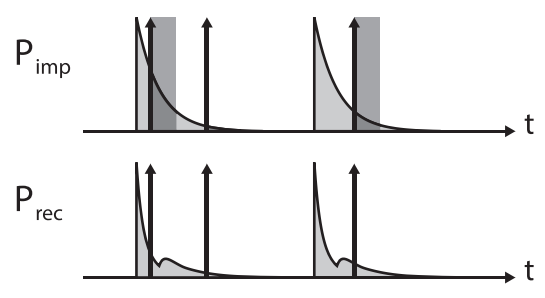

b)

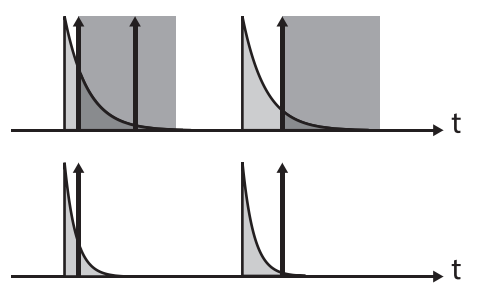

FIG. 3. Effect of a different duration of the detector dead time. In the top row, exponential pulses represent $P_{\text {imp }}(t)$ and arrows show the arrival time of impinging photons. In the bottom row, the shape of the average recording probability is shown, along with the recorded photons. In the first case (a), the dead time covers only a part of the fluorescence pulse, so more than one photon can be recorded in each period. It can be shown that a short dead time leads to a lower recording probability in the central part of the pulse. ${ }^{6}$ In the second case (b), the dead time covers the entire signal pulse, so only one photon per period can be detected and the classic pileup model applies. 
Conversely, if the dead time surpasses the duration of the fluorescence pulse, only one photon can be recorded in each period, thus resulting in classic-pileup distortion, as illustrated in Fig. 3(b). The result is a negative plateau in the curves of Fig. 2, which is well approximated by (2). When $T_{\text {dead }}$ is higher than a laser period ( $\left.T_{\text {dead }}>T_{\text {laser }}\right)$, photons recorded in a cycle generate a dead time which also influences the subsequent period. The effect is a cyclic behavior of $E$, which tends to zero when $T_{\text {dead }}$ approaches an integer multiple of $T_{\text {laser }}$.

Given the simulation result reported in Fig. 2, it is evident that the highest distortion is reached when the system operates in the pileup regime [Fig. 3(b)], as can be inferred from the high absolute value of $E$ in the plateau region. The extension of this region depends on the value of the time constant. In fact, if the pulse duration is much shorter than the laser period, a large range of $T_{\text {dead }}$ values permit to mask the whole pulse [see Fig. 3(b)], so $E$, expressed as a function of $T_{\text {dead }}$, features a large pileup plateau. Conversely, by increasing $\tau$, plateaus tend to disappear and the absolute value of $E$ reduces. It follows that low values of the time constant correspond to higher distortion. In order to consider a worst-case scenario, all the results shown in the following refer to a relatively low $\tau$ of $1 \mathrm{~ns}$.

\section{B. Counting efficiency}

As discussed above, the counting loss due to the detector dead time gives rise to a periodic distortion pattern as a function of the dead time value. It follows that the system can be operated with zero distortion exploiting a dead time equal to any integer multiple of the laser period. Nevertheless, by increasing the dead time, the average number of photons that the system records in a period, $P_{r e c}$, decreases. Starting from the same simulation used to evaluate $E$ in Fig. 2, $P_{\text {rec }}$ has been computed as the total number of recorded events divided by the total number of periods. The result is shown in Fig. 4.

Here the average number of recorded photons per period decreases in discrete steps due to the pulsed nature of the signal. In general, if no dead time is present, every photon impinging on the detector is collected in the histogram, so $P_{\text {rec }}$ is equal to $P$. By increasing $T_{\text {dead }}$, a photon impinging during the initial part of the period masks a part of the luminous signal [see Fig. 3(a)], so $P_{\text {rec }}$ decreases. This situation holds until the duration of the dead time is sufficiently high to cover the entire fluorescence pulse. At this point, a further increase of $T_{\text {dead }}$ within the laser period does not lead to an efficiency reduction, so $P_{r e c}$, expressed as a function of $T_{\text {dead }}$, features a plateau, which is well described by the classic pileup model,, 5 as shown in Fig. 3(b). When the dead time approaches the duration of the excitation period (that is $T_{\text {dead }}=12.5 \mathrm{~ns}$ in the simulation of Fig. 4), a photon impinging during a cycle is able to cause counting loss in the subsequent period. As a result, $P_{\text {rec }}$ decreased down to a second plateau. The same holds for $T_{\text {dead }}$ equal to any integer multiple of the laser period.

Considering the simple case of constant illumination of the detector over time, it is possible to obtain a closed-form expression for the counting efficiency. In fact, when photons are uniformly distributed over time, the counting efficiency $\left(\eta=P_{\text {rec }} / P_{\text {imp }}\right)$ is equal to the fraction of the measurement time during which the system is able to record photons, that is, $1-P_{\text {rec }} \cdot T_{\text {dead }} / T_{\text {laser }}$, where $T_{\text {dead }}$ is the detector dead time. In this scenario, $P_{\text {rec }}$ can be expressed as follows:

$$
P_{\text {rec }}=\frac{P}{1+P \cdot \frac{T_{\text {dead }}}{T_{\text {laser }}}} .
$$

This result is shown in Fig. 4 for $P$ equal to 1, along with the curve obtained for a pulsed illumination. It is evident that the two curves follow the same trend.

It is worth highlighting that the situation described in Fig. 4 for periodic illumination is valid only if the time constant of the fluorescence pulses is far lower than the laser period. Conversely, if the pulse width is comparable to the excitation period, the measurement speed approaches the model described in (5) and plateaus tend to disappear. In any case, the general trend expressed in (5) is confirmed.

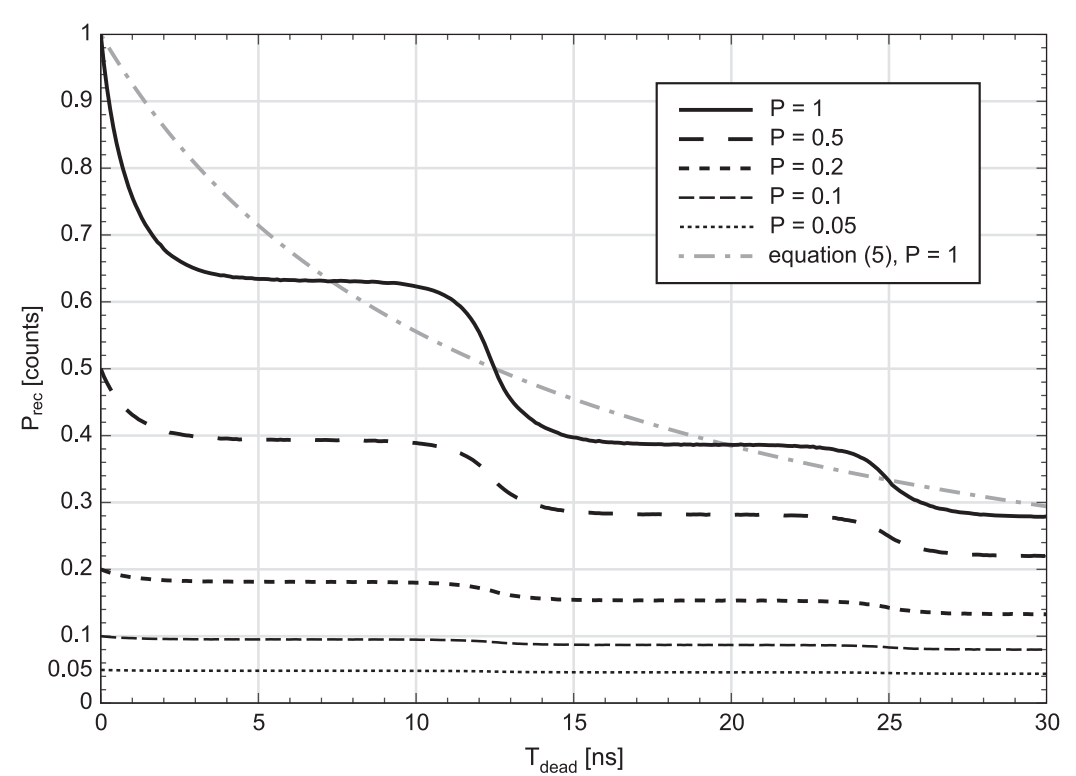

FIG. 4. Average number of recorded events in a period, $P_{r e c}$, obtained by the numerical simulation of the counting loss mechanism of Fig. 1. The result is shown as a function of the dead time $T_{\text {dead }}$ for different values of $P$, that is, the average number of impinging photons per excitation cycle. The simulation considers a pulsed fluorescence signal with a decay time constant equal to $1 \mathrm{~ns}$ and a repetition rate equal to $80 \mathrm{MHz} . P_{\text {rec }}$ in the case of constant illumination is also reported for $P$ equal to 1 photon per period, as expressed in (5). 


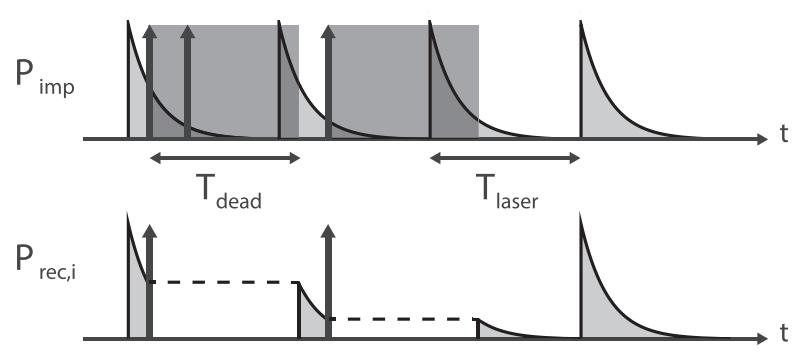

FIG. 5. Effect of a detector dead time equal to one excitation period ( $T_{\text {dead }}$ $=T_{\text {laser }}$ ). On the top, exponential pulses represent the impinging probability $P_{\text {imp }}(t)$, while the instantaneous recording probability $P_{r e c, i}(t)$ is shown in the bottom for each period. In the ideal case of $T_{\text {dead }}$ equal to zero, $P_{r e c, i}(t)$ is equal to $P_{\text {imp }}(t)$. Here the measure lasts for 4 laser cycles, but two periods remain blind due to the dead time. Nevertheless, this situation does not involve any distortion of the recorded curve. In fact, every time the system enters a dead time, it returns active starting from the same value of $P_{r e c, i}(t)$. It follows that, concerning distortion, the situation is equivalent to consider no dead time and an effective measurement time of two periods.

\section{A better insight into dead time optimization}

Starting from numerical simulations, it has been shown that a TCSPC acquisition channel can work at high speed with zero distortion, providing that the dead time of the detector is matched to an integer multiple of the excitation rate and an extremely fast conversion electronics is used.

It is possible to provide a better insight of this effect, starting from Fig. 5, where a dead time equal to one excitation period is considered. In this case, each time the system records a photon at time-instant $t$ within the period, it enters a dead time, which ends at time $t$ within the subsequent period. Concerning distortion, this situation is equivalent to consider a system with zero dead time, thus resulting in a recorded histogram proportional to the signal pulse. This result can be also mathematically derived. The average probability $P_{\text {rec }}(t)$ to record a photon at time $t$ within a generic laser period is given by the product between the impinging probability $P_{\text {imp }}(t)$ and the probability that the system is able to detect events at time $t$. As can be inferred from Fig. 1, the latter is equal to the probability that no photon is recorded within an interval of duration $T_{\text {dead }}$ before time $t$. This result can be evaluated as the integral of $P_{\text {rec }}(t)$ between $t-T_{\text {dead }}$ and $t$, considering the periodic behavior of the average recording probability,

$$
P_{\text {rec }}(t)=P_{\text {imp }}(t) \cdot\left[1-\int_{t-T_{\text {dead }}}^{t} \sum_{i=-\infty}^{\infty} P_{\text {rec }}\left(t^{\prime}+i \cdot T_{\text {laser }}\right) \cdot d t^{\prime}\right] .
$$

In the simple case of dead time equal to an integer number $n$ of laser periods $\left(T_{\text {dead }}=n \cdot T_{\text {laser }}\right)$, the integral of the periodic recording probability is equal to the area of a single pulse multiplied by $n$. As a result, Eq. (6) can be simplified as follows:

$$
P_{r e c}(t)=P_{\text {imp }}(t) \cdot\left[1-n \cdot \int_{0}^{T_{\text {laser }}} P_{r e c}\left(t^{\prime}\right) \cdot d t^{\prime}\right] .
$$

It is evident that the integral part does not depend on $t$, so the ratio between $P_{\text {rec }}(t)$ and $P_{\text {imp }}(t)$ is a constant. It follows that no distortion is affecting the experiment. It is worth noting that this result applies regardless of the shape of the luminous signal, for instance, even when more than a fluorescence time constant is present.

Starting from (7), it is also possible to evaluate the measurement speed of the system. In fact, the factor of proportionality between the recorded curve and the signal represents the counting efficiency $\eta$. As a result,

$$
\eta=1-n \cdot \int_{0}^{T_{\text {laser }}} P_{\text {rec }}\left(t^{\prime}\right) \cdot d t^{\prime}=1-\eta \cdot n \cdot \int_{0}^{T_{\text {laser }}} P_{\text {imp }}\left(t^{\prime}\right) \cdot d t^{\prime}
$$

since $P_{\text {rec }}(t)$ is equal to $\eta \cdot P_{\text {imp }}(t)$.

It is well known that the area of the impinging probability $P_{\text {imp }}(t)$ over a period is equal to the mean number of photons impinging on the detector over a period that is $P$; hence the average number of recorded photons in a period, $P_{r e c}$, can be expressed as follows:

$$
P_{\text {rec }}=\eta \cdot P=\frac{P}{1+n \cdot P}=\frac{P}{1+P \cdot \frac{T_{\text {dead }}}{T_{\text {laser }}}} .
$$

It is worth noting that this result is equivalent to the one expressed by (5), which was derived for a constant illumination of the detector.

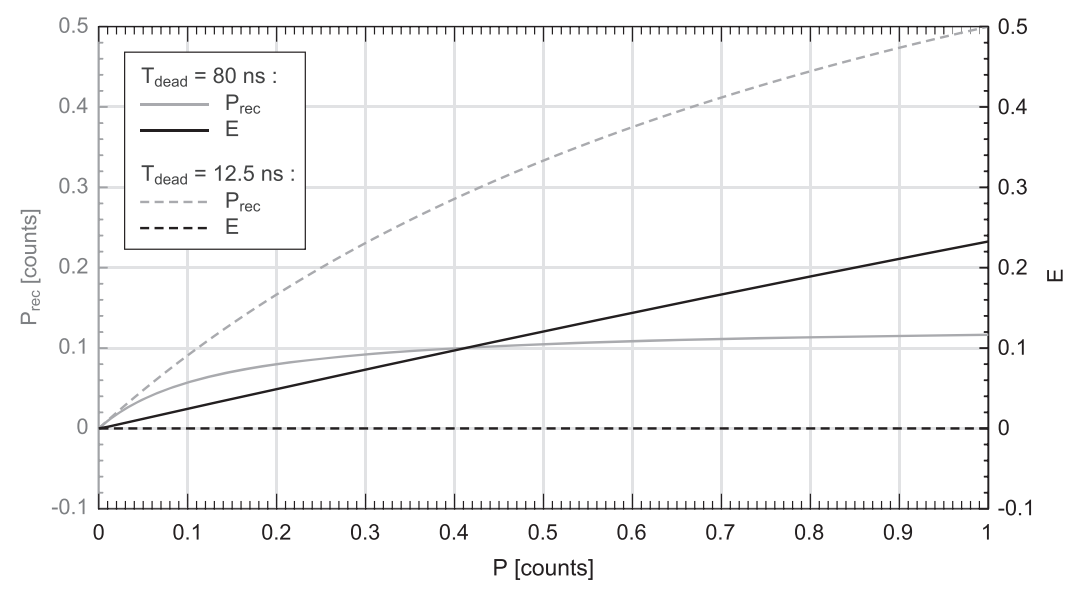

FIG. 6. Comparison between the solution proposed in Fig. 5, with $T_{\text {dead }}=T_{\text {laser }}=12.5 \mathrm{~ns}$ (dashed lines), and a typical TCSPC acquisition system with an electronics dead time of $80 \mathrm{~ns}$ (continuous lines). The fractional estimation error $E$ and the average number of recorded photons in a period $P_{\text {rec }}$ are reported for both architectures as a function of the average number of impinging photons in a period, $P$. 
Equation (9) shows that the measurement speed (proportional to $P_{r e c}$ ) increases with the power of the luminous signal. At the same time, the system features zero distortion, regardless of the value of $P$, thus allowing unprecedented high-speed operation in TCSPC experiments using a single acquisition channel. In Fig. 6, both $E$ and $P_{\text {rec }}$ resulting from the optimum condition depicted in Fig. 5 are compared with the performance of a system limited by an electronics dead time equal to $80 \mathrm{~ns}$, which is a typical value for commercial TCSPC modules. ${ }^{22}$ It is evident that the approach of Fig. 5 (dashed lines) leads to both higher speed and zero distortion.

\section{NON-IDEALITIES OF A PRACTICAL IMPLEMENTATION}

It has been demonstrated how a detector dead time equal to an integer number $n$ of excitation periods allows the recording of non-distorted histograms in TCSPC experiments, irrespective of the average impinging rate. In order to exploit this optimum working condition, a fine tuning of the detector dead time is needed. At the same time, it is clear that the highest counting efficiency is reached for the minimum value of $n$, that is, for a dead time equal to one laser period, as expressed in (9).

In recent years, Active Quenching Circuits (AQCs) for Single Photon Avalanche Diodes (SPADs) featuring $T_{\text {dead }}$ in the order of $10 \mathrm{~ns}^{23-26}$ have been proposed, paving the way to dead time optimization, even when a relatively high excitation rate of $80 \mathrm{MHz}$ is considered. For instance, in 2016, we proposed a very versatile AQC, able to drive the external SPADs of different technologies and able to provide a tunable dead time down to $10 \mathrm{~ns}$ with a $50-\mu \mathrm{m}$ custom-technology SPAD detector. ${ }^{18,19}$

In any case, regardless of the practical implementation of the proposed solution, some non-idealities of the setup can impair the results shown in Fig. 6. For instance, considering an active quenching of the detector, the duration of the quenching phase is unavoidably affected by a jitter of the control logic, so the duration of the dead time slightly varies during the measurement.

Even worse, at the end of the dead time, the initial bias condition of the detector is restored. It is evident that this operation requires a finite reset time to be carried out, so the recording probability does not feature a step-like transition at the end of the dead time. In this scenario, the system is able to detect photons also during the final part of the dead time.

In order to evaluate the impact of practical implementation of the solution on both distortion and measurement speed, the non-idealities of the system have been included in numerical simulations, considering reasonable values for jitter and reset duration. In particular, the AQC proposed in Ref. 18 has been considered as an example to perform some numerical simulations.

\section{A. Dead time jitter}

The effect of a random fluctuation of the dead time during a TCSPC measurement was simulated, starting from a
Poisson distribution of photons with different values of decay time constant. In this case, each time an event is detected, the system remains blind for a dead time, whose duration is randomly drawn from a Gaussian distribution with mean equal to one excitation period. For recently proposed AQCs, a dead time jitter in the order of $100 \mathrm{ps}$ r.m.s. has to be considered. For instance, we measured about $90 \mathrm{ps}$ r.m.s. considering the quenching circuit presented in Ref. 18, connected to the anode of a $50-\mu \mathrm{m}$ custom SPAD.

Considering an average dead time equal to $12.5 \mathrm{~ns}$ and a reasonable value of the jitter, ranging from 0 to 200 ps r.m.s., we simulated the fractional estimation error $E$ for different values of $\tau$ between 1 and $4 \mathrm{~ns}$ and $P$ ranging from 0 to 2 (that is 40 times higher than the pileup limit). In all these cases, $E$ resulted limited below $1 \%$, even when the lowest time constant $(\tau=1 \mathrm{~ns})$ and the highest impinging rate $(P=2)$ were considered.

We also verified that the measurement speed still follows the trend expressed in (9), even when considering a dead time jitter around $100 \mathrm{ps}$.

\section{B. Photons detected during the reset phase}

Considering a practical implementation of the quenching circuit, like the one reported in Ref. 18, a finite time $T_{\text {reset }}$ is needed to recover the initial bias condition of the detector at the end of the dead time. As a result, the probability to detect a photon after an event has been recorded does not experience a step-like transition, like in the simple model of Fig. 5, but photons impinging during the last part of the dead time have a non-zero probability to be detected. The situation is shown in Fig. 7.

In this case, each time a photon is detected during the reset time, an event is added to the histogram; so the system moves away from the optimum condition since the equivalent dead time associated with the recorded events is less than one excitation period. The result is a distortion of the recorded histogram.

In order to allow an estimation of the distortion, simulations have been performed considering a realistic transition of the detection probability at the end of the dead time, which typically lasts for some nanoseconds. ${ }^{18,19,23-26}$ For instance,

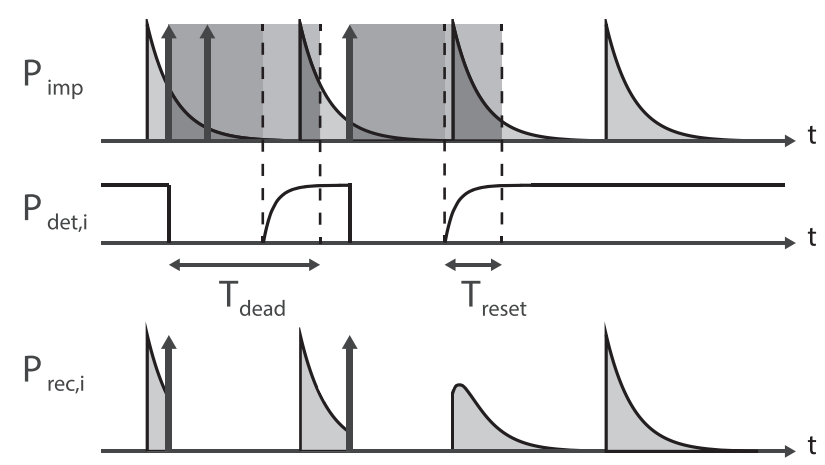

FIG. 7. Effect of a finite transition of the detection probability at the end of the dead time. During the reset phase, the instantaneous detection probability $P_{\text {det }, i}$ increases gradually up to the maximum value, so the instantaneous recording probability is not zero during the dead time. The result is a distortion of the recorded histogram. 


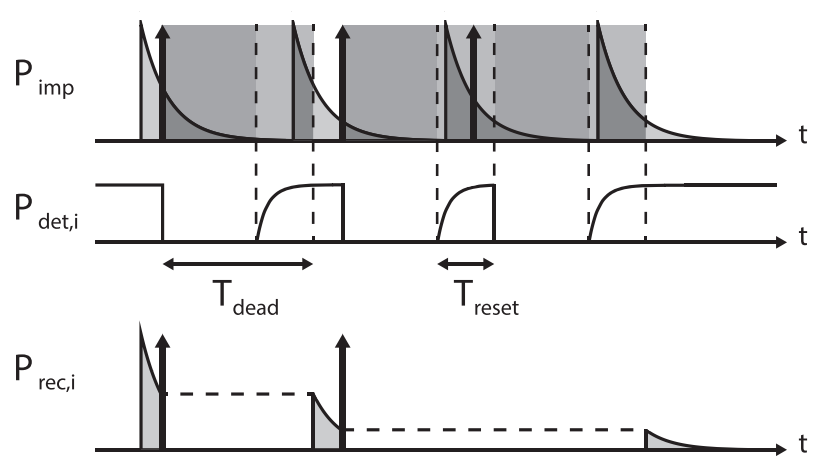

FIG. 8. The detection probability features a gradual transition during the reset time, so impinging photons have a probability $P_{d e t, i}$ to trigger an avalanche in the detector during the last part of the dead time. Then the system enters in a new dead time starting from the end of the previous one, thus resulting in an equivalent dead time lasting for two periods. Nevertheless, events are not registered during the reset phase. It follows that the ideal shape of the recording probability is distributed among many periods, like in the optimum condition depicted in Fig. 5; so no distortion is generated in the recorded histogram.

we estimated a gradual transition of $P_{d e t}$ that completes in about $4 \mathrm{~ns}$, considering the AQC described in Ref. 18 coupled to a custom SPAD. In particular, $P_{\text {det }}$ was calculated combining the Photon Detection Efficiency (PDE) of the SPAD, expressed as a function of the voltage applied to the device, with the voltage transition at the SPAD anode during the reset phase. The actual shape of the detection probability has been included in numerical simulations and $E$ has been extracted for different values of $T_{\text {dead }}, P$, and $\tau$. Simulations revealed that dead time optimization is no more feasible in the scenario depicted in Fig. 8. In fact, we could not find a value of $T_{\text {dead }}$, which guarantees zero distortion, regardless of the values of $P$ and $\tau$. As a result, the overall distortion still increases with the signal power, thus demanding for low-rate measurements.

To surpass this major issue, we further improved the proposed technique: in the following, we show that the distortion can be reduced to negligible levels even when $P_{\text {rec }}$ features a finite transition at the end of the dead time, providing that events detected during the reset phase are not included in the histogram. In this scenario, each time a photon triggers an avalanche during the dead time, no distortion is involved, but the system remains blind for an additional time interval, so $P_{r e c}$ drops below the value reported in (9).

In Fig. 8, the effect of the proposed technique is shown. When a photon is detected during the end of the dead time $\left(T_{\text {reset }}\right)$, the system remains blind for an additional $T_{\text {dead }}$. The result is an equivalent dead time that lasts for two laser periods. It is clear that this approach leads to a variable dead time, which depends on the presence of photons detected during the reset time. Nevertheless, $E$ is kept to zero since the dead time is always an integer multiple of $T_{\text {laser }}$.

A simulation of the distortion obtained with the proposed approach is shown in Fig. 9, where a realistic transition of the recording probability has been considered, which lasts for a duration $T_{\text {reset }}$ equal to $4 \mathrm{~ns}$. It is evident that the system features no distortion if $T_{\text {dead }}=T_{\text {laser }}$, regardless of the average number of impinging photons in one period, $P$.

The average number of recorded events in one period, which is proportional to the measurement speed, has been extracted starting from the same simulation. The result is shown in Fig. 10 (dotted curve) and compared with (9). A theoretical curve for the simulated system is also reported, which is described in detail later in Sec. IV C. As expected, the curves approach the same values at low frequencies, where the probability to detect a photon during a reset phase is negligible. Then, increasing the impinging frequency, the simulated curve starts decreasing down to 0 . In fact, if the impinging frequency is much higher than one photon per period (that is $P>>1$ ), the probability to trigger a new dead time during a reset phase becomes relevant and the overall dead time can increase up to the whole measurement duration.

As can be inferred from Fig. 10, the maximum of the measurement speed is reached when $P$ approaches one photon per period, corresponding to a recording frequency of almost $40 \%$ of the laser rate, which is much higher than a typical pileup-limited detection rate, equal to $5 \%$ of the excitation period. So a system working like in Fig. 8 leads to a remarkable gain of a factor 8 in the measurement speed.

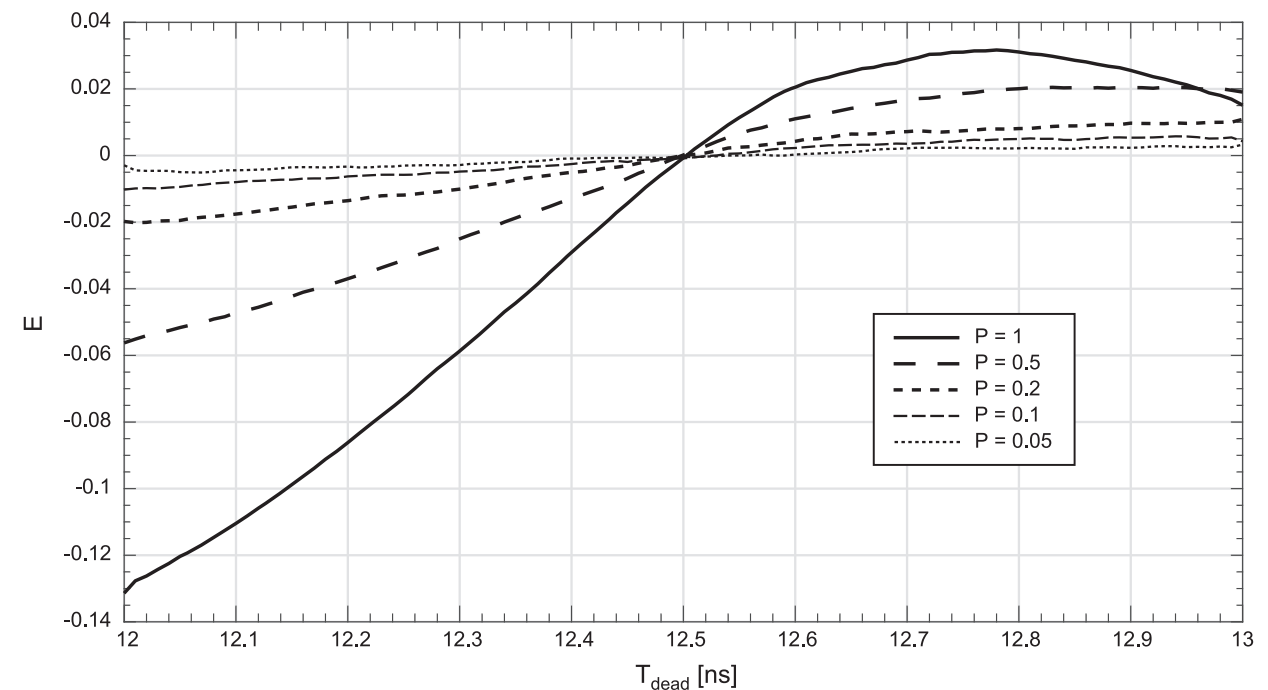

FIG. 9. Fractional error in the estimation of the time constant, $E$, as a function of the detector dead time $T_{\text {dead }}$ in the scenario of Fig. 5, considering fluorescence pulses with $\tau$ equal to $1 \mathrm{~ns}$ and $80 \mathrm{MHz}$ repetition rate. During the reset time, which lasts for $4 \mathrm{~ns}$, the detection probability increases gradually up to 1 , but photons detected during this phase are discarded. It is evident that $E$ is 0 if $T_{\text {dead }}$ is equal to one excitation period, regardless of the value of $P$, that is, the average number of impinging photons in one period. 


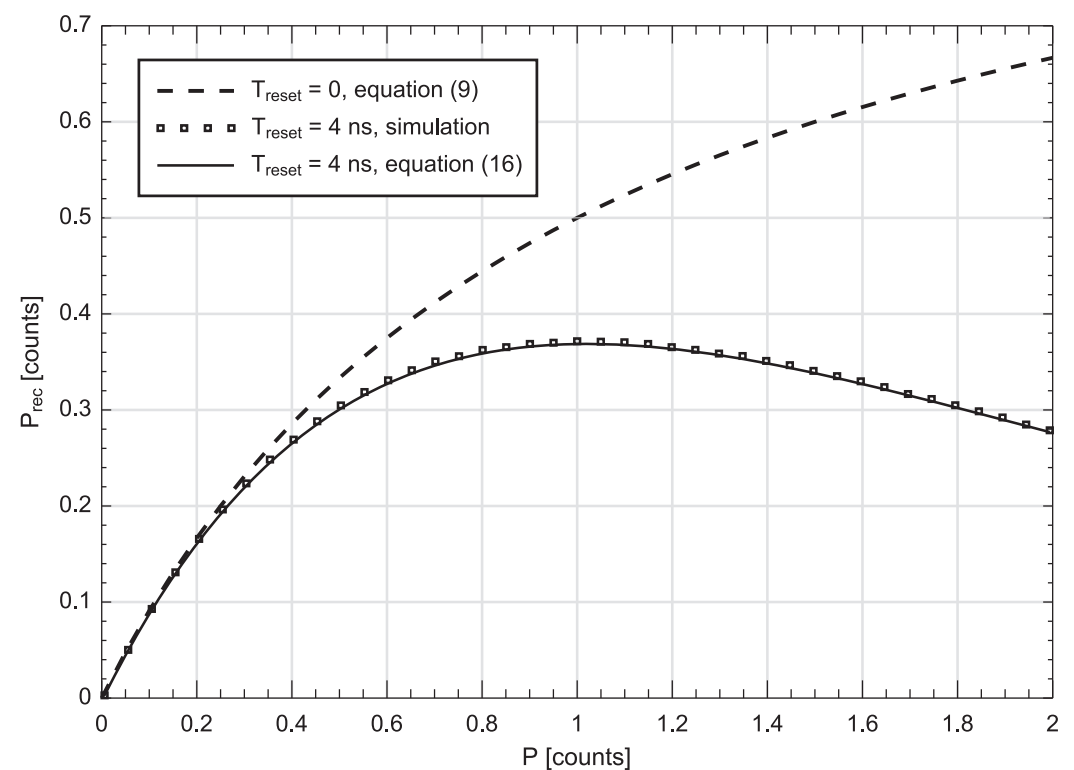

FIG. 10. Comparison between the average number of recorded events in one period in the optimum case of zero reset time, as expressed in (9), and using the approach proposed in Fig. 8, where $T_{\text {reset }}$ is equal to $4 \mathrm{~ns}$. In the latter, the curve reaches a maximum at an impinging rate $P$ equal to one photon per period. In this case, the maximum $P_{\text {rec }}$ is equal to $37 \%$ of the excitation rate, which is well above the pileup limit. The theoretical formula of $P_{\text {rec }}$ for the simulated system is also reported, which is described by Eq. (16). A time constant $\tau$ equal to $1 \mathrm{~ns}$ has been considered for all the curves.
It is worth highlighting that a faster voltage transition during the reset phase can lead to even better results since the recording probability tends to approach the curve expressed in (9). For instance, a maximum recording probability equal to $45 \%$ at $P$ around 1.4 has been obtained for $T_{\text {reset }}=1 \mathrm{~ns}$.

\section{Analytical derivation of the measurement speed}

Considering the situation illustrated in Fig. 8, a closedform expression can be derived for the counting efficiency, $\eta$. Given a long measurement time, $T_{\text {measure }}$, the system is blind for an integer number $N$ of intervals of duration $T_{\text {dead }}$, which is equal to one excitation period $\left(T_{\text {dead }}=T_{\text {laser }}\right)$. In this scenario, the efficiency can be expressed as the fraction of time when the system is able to record photons, that is,

$$
\eta=1-\frac{N \cdot T_{\text {laser }}}{T_{\text {measure }}}=1-\frac{T_{\text {dead }, \text { TOT }}}{T_{\text {measure }}}
$$

where $N$ represents the number of times an avalanche is triggered during the measurement, while $T_{\text {dead }, \text { TOT }}$ is the sum of the intervals where the system is blind.

Referring to Fig. 8, each time a photon is detected at time $t$ within the excitation period, a reset time starts at time $t+T_{\text {laser }}-T_{\text {reset }}$ and ends at time $t+T_{\text {laser }}$. The average number of photons which are able to trigger an avalanche during this interval, $P_{\text {det, reset }}$, can be computed as the integral of the probability that an avalanche is triggered at time $t^{\prime}$, with $t^{\prime}$ ranging from $t+T_{\text {laser }}-T_{\text {reset }}$ to $t+T_{\text {laser }}$. The probability to observe an avalanche at time $t^{\prime}$ is given by the product between the detection probability $P_{\text {det }}\left(t^{\prime}\right)$ (which features a finite transition during the reset phase) and the average number of impinging photons in an infinitesimal-sized interval $d t^{\prime}$ centered at $t^{\prime}$. Considering the periodic behavior of the impinging probability,

$$
P_{\text {det, reset }}(t)=\int_{t+T_{\text {laser }}-T_{\text {reset }}}^{t+T_{\text {laser }}} P_{\text {det }}\left(t^{\prime}\right) \cdot \sum_{i=-\infty}^{\infty} P_{\text {imp }}\left(t^{\prime}+i \cdot T_{\text {laser }}\right) \cdot d t^{\prime} .
$$

As can be inferred from Fig. 8, Eq. (11) can be rearranged as follows:

$$
P_{\text {det, reset }}(t)=\left\{\begin{array}{ll}
\int_{t+T_{\text {laser }}-T_{\text {reset }}}^{T_{\text {laser }}} P_{\text {det }}\left(t^{\prime}\right) \cdot P_{0} \cdot e^{-t^{\prime} / \tau} \cdot d t^{\prime}+\int_{0}^{t} P_{\text {det }}\left(t^{\prime}\right) \cdot P_{0} \cdot e^{-t^{\prime} / \tau} d t^{\prime}, t \leq T_{\text {reset }} \\
\int_{t-T_{\text {reset }}}^{t} P_{\text {det }}\left(t^{\prime}\right) \cdot P_{0} \cdot e^{-t^{\prime} / \tau} \cdot d t^{\prime}, & t>T_{\text {reset }}
\end{array},\right.
$$

where the first integral can be neglected if the time constant $\tau$ is far lower than $T_{\text {laser }}-T_{\text {reset }}$.

Given a Poisson distribution of the impinging photons, the probability that no photon triggers an avalanche during the reset phase is equal to $\exp \left(-P_{\text {det, reset }}\right)$, while the probability that an avalanche is triggered during the reset time is equal to the probability that at least one photon is detected during the reset phase, which is equal to $1-\exp \left(-P_{\text {det, reset }}\right)$. 
As stated before, a photon recorded at time $t$ leads to an effective dead time which depends on the presence of photons recorded during the reset time. For instance, the effective dead time has a duration $T_{\text {laser }}$ if no photon is detected within the reset phase. Conversely, if an avalanche is triggered during the reset phase, a new dead time of duration $T_{\text {laser }}$ starts in sync with the end of the first dead time (see Fig. 10), involving a new reset phase. If no photon is detected during this phase, the effective dead time has a duration $2 \cdot T_{\text {laser }}$. In general, the probability that the dead time associated with a photon recorded at time $t$ is equal to $n \cdot T_{\text {laser }}$ is equal to $\left[1-\exp \left(-P_{\text {det,reset }}\right)\right]^{n-1} \cdot \exp \left(-P_{\text {det,reset }}\right)$, that is, the probability to observe an avalanche in $n-1$ subsequent reset phases and no avalanche in the $\mathrm{n}$-th dead period. It follows that the average duration of the dead time associated with a photon detected at time $t$ can be expressed as the probability-weighted mean of all possible dead time durations, that is, $n \cdot T_{\text {laser }}$, with $n$ ranging from 1 to infinite,

$$
\begin{aligned}
T_{\text {dead }, \text { av }}(t) & =\sum_{n=1}^{\infty} n \cdot T_{\text {laser }} \cdot e^{-P_{\text {det, reset }}(t)} \cdot\left(1-e^{-P_{\text {det, reset }}(t)}\right)^{n-1} \\
& =T_{\text {laser }} \cdot e^{P_{\text {det, }, \text { reset }}(t)} .
\end{aligned}
$$

The probability that a photon is recorded at a time instant $t$ within a laser period can be expressed as the product between the probability density function $P_{\text {rec }}(t)$ and the infinitesimalsized interval $d t$. Then, the total dead time in a measure, $T_{\text {dead }, T O T}$, can be computed as the integral over the measure of the probability to record a photon at time $t$, multiplied by the average dead time $T_{\text {dead ,av }}(t)$. Given the periodic behavior of the experiment, the same result can be obtained integrating over a period and multiplying by the number of periods, that is, $T_{\text {measure }} / T_{\text {laser }}$,

$$
\begin{aligned}
T_{\text {dead }, \text { TOT }} & =\frac{T_{\text {measure }}}{T_{\text {laser }}} \cdot \int_{0}^{T_{\text {laser }}} P_{\text {rec }}(t) \cdot d t \cdot T_{\text {laser }} \cdot e^{P_{\text {det, reset }}(t)} \\
& \approx T_{\text {measure }} \cdot \int_{0}^{\infty} \eta \cdot \frac{P}{\tau} \cdot e^{-t / \tau} \cdot d t \cdot e^{P_{\text {det, reset }}(t)}, \quad
\end{aligned}
$$

where $P_{\text {rec }}(t)$, equal to $\eta \cdot P_{i m p}(t)$, has been expressed using (1). It is worth noting that $P_{0}$ is equal to $P / \tau$ only if the width of the luminous pulse is shorter than the duration of a repetition period (i.e., $\tau<<T_{\text {laser }}$ ). Under the same conditions, the integral has been extended from 0 to infinite.

It is evident from (11) that $P_{\text {det,reset }}(t)$ depends on the shape of the detection probability during the reset phase, which is strongly dependent on the implementation of the quenching circuit. In order to extract a useful expression of the counting efficiency, independent from the practical implementation of the experiment, it is possible to impose $P_{d e t}\left(t^{\prime}\right)$ equal to 1 during the whole reset phase. The result is an overestimation of the probability that a photon triggers an avalanche during the reset phase, thus leading to a conservative estimation of the efficiency. Considering $\tau$ much lower than $T_{\text {laser }}-T_{\text {reset }}$ and $P_{d e t}\left(t^{\prime}\right)$ equal to 1 , the combination of (12) and (14) leads to the following result:

$$
\begin{aligned}
T_{\text {dead }, \text { TOT }}= & T_{\text {measure }} \cdot \eta \cdot\left[\int_{0}^{T_{\text {reset }}} \frac{P}{\tau} \cdot e^{-t / \tau} \cdot \exp \left[P \cdot\left(1-e^{-t / \tau}\right)\right] \cdot d t\right. \\
& \left.+\int_{T_{\text {reset }}}^{\infty} \frac{P}{\tau} \cdot e^{-t / \tau} \cdot \exp \left[P \cdot\left(e^{T_{\text {reset }} / \tau}-1\right) \cdot\left(e^{-t / \tau}\right)\right] \cdot d t\right] .
\end{aligned}
$$

Rearranging (10) and (15), the expression of the counting efficiency $\eta$ is found. As a result,

$$
P_{\text {rec }}=\eta \cdot P=\frac{1}{\exp \left[P \cdot\left(1-e^{-T_{\text {reset }} / \tau}\right)\right]+\frac{\exp \left[P \cdot\left(1-e^{-T_{\text {reset }} / \tau}\right)\right]-1}{e^{T_{\text {reset }} / \tau}-1}} \cdot P .
$$

Expression (16) is reported along with the simulated results in Fig. 10. In the simulation, the time constant $\tau$ is $1 \mathrm{~ns}$, while the reset time $T_{\text {reset }}$ is $4 \mathrm{~ns}$, which is much higher than $\tau$, so the counting efficiency expressed in (16) can be approximated with $\exp (-P)$. It follows that the average number of recorded photons, $P_{r e c}$, is equal to $P \cdot \exp (-P)$, so the maximum $P_{\text {rec }}$ is reached at $P$ equal to 1 and is equal to $1 / e$, that is, about 0.37 . This result is in accordance with the simulation of Fig. 10, which was performed considering a realistic transition of the detection probability during the reset time.

\section{Combined effect of jitter and finite reset transition}

Considering the system described in Fig. 8, the presence of a gradual transition of the detection probability during the reset phase does not introduce any distortion but sets only a limit to the maximum measurement speed. As a result, the only source of distortion comes from the dead time jitter, as discussed earlier.

The combined effect of both the dead time jitter and a finite transition of the recording probability has been included in simulations, considering a dead time jitter around $100 \mathrm{ps}$ r.m.s. and $T_{\text {reset }}$ equal to some nanoseconds. The result was an estimation error, which is still limited well below $1 \%$. It follows that a system working as described in Fig. 8 is effectively able to work at high frequency, without any significant distortion of the recorded curve.

\section{CONCLUSIONS}

Nowadays the measurement speed of conventional TCSPC conversion channels is limited to some percent of the excitation frequency in order to avoid distortions of the recorded histogram.

In this paper, we present a novel approach to speed up TCSPC measurements, without impairing the performance of the system in terms of linearity. In particular, we have theoretically proved how the exploitation of a fast conversion electronics, along with a detector, with a dead time matched to the excitation period enables a gain of almost an order of magnitude in the speed of TCSPC measurements providing, at the same time, almost-zero distortion. 
It is worth highlighting that our solution can be easily extended to a multichannel approach to optimize its performance in terms of counting capability and linearity.

${ }^{1}$ W. Becker, J. Microsc. 247, 119 (2012).

${ }^{2}$ H. Wallrabe and A. Periasamy, Curr. Opin. Biotechnol. 16, 19 (2005).

${ }^{3}$ L. Kelbauskas and W. Dietel, Photochem. Photobiol. 76, 686 (2002).

${ }^{4}$ K. Okabe, N. Inada, C. Gota, Y. Harada, T. Funatsu, and S. Uchiyama, Nat. Commun. 3, 705 (2012).

${ }^{5} \mathrm{~W}$. Becker, Advanced Time-Correlated Single Photon Counting Techniques (Springer Science \& Business Media, Berlin, 2005).

${ }^{6} \mathrm{~J}$. Artl, D. Tyndall, B. R. Rae, D. D.-U. Li, J. A. Richardson, and R. K. Henderson, Rev. Sci. Instrum. 84, 103105 (2013).

${ }^{7}$ A. S. Yousif and J. W. Haslett, IEEE Trans. Nucl. Sci. 54, 1574 (2007).

${ }^{8}$ N. A. W. Dutton, S. Gnecchi, L. Parmesan, A. J. Holmes, B. Rae, L. A. Grant, and R. K. Henderson, Dig. Tech. Pap. - IEEE Int. Solid-State Circuits Conf. 58, 204 (2015).

${ }^{9}$ D. Tyndall, B. Rae, D. Li, J. Richardson, J. Arlt, and R. Henderson, Dig. Tech. Pap. - IEEE Int. Solid-State Circuits Conf. 55, 122 (2012).

${ }^{10}$ P. Peronio, G. Acconcia, I. Rech, and M. Ghioni, Rev. Sci. Instrum. 86, 113101 (2015).

${ }^{11}$ S. Orthaus-Mueller, B. Kraemer, A. Tannert, T. Roehlicke, M. Wahl, H.-J. Rahn, F. Koberling, and R. Erdmann, Proc. SPIE 10069, 1006919 (2017).

${ }^{12}$ N. Krstaji, J. Levitt, S. Poland, S. Ameer-beg, and R. Henderson, Opt. Express 23, 5653 (2015).

${ }^{13}$ C. Veerappan, J. Richardson, R. Walker, D. U. Li, M. W. Fishburn, Y. Maruyama, D. Stoppa, F. Borghetti, M. Gersbach, R. K. Henderson, and E. Charbon, Dig. Tech. Pap. - IEEE Int. Solid-State Circuits Conf. 312, 312-314 (2011).
${ }^{14}$ F. Villa, R. Lussana, D. Bronzi, S. Tisa, A. Tosi, F. Zappa, A. Dalla Mora, D. Contini, D. Durini, S. Weyers, and W. Brockherde, IEEE J. Sel. Top. Quantum Electron. 20, 364 (2014).

${ }^{15}$ L. Parmesan, N. A. Dutton, N. J. Calder, N. Krstajic, A. J. Holmes, L. A. Grant, and R. K. Henderson, "A $256 \times 256$ SPAD array with in-pixel time to amplitude conversion for fluorescence lifetime imaging microscopy," in International Image Sensor Workshop, Vaals, Netherlands (2015), Vol. 900, pp. 8-11.

${ }^{16}$ R. M. Field, S. Realov, and K. L. Shepard, IEEE J. Solid-State Circuits 49, 867 (2014).

${ }^{17}$ A. Cominelli, G. Acconcia, P. Peronio, I. Rech, and M. Ghioni, IEEE Photonics J. 9, 1 (2017).

${ }^{18}$ G. Acconcia, I. Rech, A. Gulinatti, and M. Ghioni, Opt. Express 24, 17819 (2016).

${ }^{19}$ G. Acconcia, I. Labanca, I. Rech, A. Gulinatti, M. Ghioni, G. Acconcia, I. Labanca, I. Rech, A. Gulinatti, and M. Ghioni, Rev. Sci. Instrum. 88, 026103 (2017)

${ }^{20}$ D. Li, R. Andrews, J. Artl, and R. Henderson, J. Biomed. Opt. 15, 046009 (2010).

${ }^{21}$ M. Köllner and J. Wolfrum, Chem. Phys. Lett. 200, 199 (1992).

${ }^{22}$ A. Giudice, R. Biasi, I. Rech, S. Marangoni, I. Labanca, G. Simmerle, M. Ghioni, and S. Cova, J. Mod. Opt. 56, 317-325 (2009).

${ }^{23} \mathrm{C}$. Niclass and M. Soga, IEEE Int. Electron Devices Meet. 340, 14.3.1-14.3.4 (2010).

${ }^{24}$ A. Eisele, R. Henderson, B. Schmidtke, T. Funk, L. Grant, J. Richardson, and W. Freude, " $185 \mathrm{MHz}$ count rate $139 \mathrm{~dB}$ dynamic range singlephoton avalanche diode with active quenching circuit in $130 \mathrm{~nm}$ CMOS technology," in Proc. Int. Image Sensor Workshop (2011), pp. 278-280.

${ }^{25}$ E. A. G. Webster, J. A. Richardson, L. A. Grant, D. Renshaw, and R. K. Henderson, IEEE Electron Device Lett. 33, 694 (2012).

${ }^{26}$ D. Bronzi, S. Tisa, F. Villa, S. Bellisai, A. Tosi, and F. Zappa, IEEE Photonics Technol. Lett. 25, 776 (2013). 